\title{
Best lower bound on the probability of a binomial exceeding its expectation
}

\author{
Iosif Pinelis \\ Department of Mathematical Sciences \\ Michigan Technological University \\ Houghton, Michigan 49931, USA \\ E-mail: ipinelis@mtu.edu
}

\begin{abstract}
Let $X$ be a random variable distributed according to the binomial distribution with parameters $n$ and $p$. It is shown that $\mathrm{P}(X>\mathrm{E} X) \geqslant 1 / 4$ if $1>p \geqslant c / n$, where $c:=\ln (4 / 3)$, the best possible constant factor.

Keywords: binomial distribution, probability inequalities, exact bounds 2010 MSC: 60E15, 62E15
\end{abstract}

\section{Summary and discussion}

Theorem 1. Let $X=X_{n, p}$ be a random variable (r.v.) with the binomial distribution with parameters $n$ and $p$. Then

$$
\mathrm{P}(X>\mathrm{E} X) \geqslant 1 / 4
$$

if

$$
1>p \geqslant c / n
$$

where

$$
c:=\ln (4 / 3)=0.28768 \ldots
$$

Under condition (2), the equality in (1) is attained only if $n=2$ and $p=1 / 2$. The constant factor $c$ in 22 is the best possible.

Complementing Theorem 1 is the following simple proposition.

Proposition 2. If $c / n \geqslant p \geqslant 0$, then $\mathrm{P}(X>\mathrm{E} X)=1-(1-p)^{n} \geqslant \max (1, b n) p$, where $b:=\left(1-e^{-c}\right) / c=0.86901 \ldots$

A very short proof of Theorem 1 will be given in Section 2, This proof is based on a monotonicity result due to Anderson and Samuels [2, which in turn follows from a more general result due to Hoeffding [7].

Preprint submitted to Statistics and Probability Letters 
A bit longer proof of Theorem 1, which may still be of interest, is relegated to the appendix. This second proof is based on a version of the Berry-Esseen bound, which takes care of the main case when $n p \geqslant 2$ and $n(1-p) \geqslant 2$, that is, when $2 \leqslant \mathrm{E} X \leqslant n-2$. The remaining cases are rather easy to deal with, since all the values of $X$ are in the set $\{0, \ldots, n\}$.

Previously it was shown [5] that, for $X$ as in Theorem 1, one has

$$
\mathrm{P}(X \geqslant \mathrm{E} X)>1 / 4
$$

if

$$
p>1 / n \text {. }
$$

Theorem 1 improves the result of [5] in two ways at once:

(i) The (optimal) constant factor $c=0.28768 \ldots$ in (2) is better than the corresponding constant factor 1 in (5). (Concerning the strictness of the inequality $\mathrm{P}(X \geqslant \mathrm{E} X)>1 / 4$ in (4), here one may recall that the inequality $\mathrm{P}(X>\mathrm{E} X) \geqslant 1 / 4$ in $(1)$ is strict unless $n=2$ and $p=1 / 2$ - in which latter case condition (5) fails to hold.)

(ii) Instead of the probability $\mathrm{P}(X \geqslant \mathrm{E} X)$ in (4), we have the (possibly) smaller probability $\mathrm{P}(X>\mathrm{E} X)$ in $(1)$.

Improvement (i) and the optimality of the constant factor $c$ are illustrated in Figure 1, showing the graphs

- $\left\{\left(p, \mathrm{P}\left(X_{n, p}>n p\right)\right): 1 / n \leqslant p<1\right\}$ (solid)

- $\left\{\left(p, \mathrm{P}\left(X_{n, p}>n p\right)\right): c / n<p \leqslant 1 / n\right\}$ (dashed, black)

- $\left\{\left(p, \mathrm{P}\left(X_{n, p}>n p\right)\right): 0<p \leqslant c / n\right\}$ (dashed, gray)

for $n=5$. This figure is similar to [5, Figure 2], where the graphs over the interval $(c / n, 1 / n]$ were dashed, too.

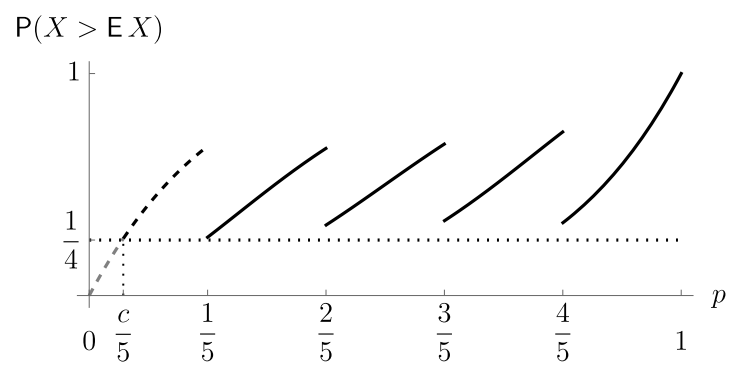

Figure 1: Graphs of $\mathrm{P}(X>\mathrm{E} X)$.

However, concerning improvement (i), one should note that the case when $c \leqslant n p<1$ - considered in the beginning of the proof of Theorem 1 - is comparatively simple. As for improvement (ii), inequality (1) follows from its 
non-strict counterpart $\mathrm{P}(X \geqslant \mathrm{E} X) \geqslant 1 / 4$ upon noting that $\mathrm{P}(X>\mathrm{E} X)=$ $\mathrm{P}\left(X_{n, p}>n p\right)$ is right-continuous in $p$ and $\mathrm{P}(X>\mathrm{E} X)=\mathrm{P}(X \geqslant \mathrm{E} X) \geqslant 1 / 4$ if $n p$ is not an integer.

So, the main distinction of the present note from [5] is perhaps that each of the two proofs of Theorem 1 given here appears to be significantly simpler than the proof in [5].

As noted in [5], inequality (4) was used several times in the machine learning literature, including [4, 14, 13, to bound the probability of the so-called relative deviation of frequencies from the corresponding probabilities for certain classes of events. Such results have applications to the so-called probablyapproximately-correct (PAC) models of machine learning; concerning PAC models, see e.g. [12, 6, 8, 1,

In [3, the non-strict version, $\mathrm{P}(X \geqslant \mathrm{E} X) \geqslant 1 / 4$, of inequality (1) was obtained, but only for large enough $n$ and $p \geqslant 2 / n$.

In [11, Lemma 13], it was shown that

$$
\mathrm{P}(X \geqslant \mathrm{E} X) \geqslant \min (p, 1 / 4)
$$

for

$$
p \in(0,1 / 2]
$$

This was used to prove a part of [11, Proposition 8]. To state that result, we need to reproduce several definitions from [11. Let $(X, Y)$ be a random vector in $\mathfrak{X} \times\{-1,1\}$, where $\mathfrak{X}$ is a Borel subset of $\mathbb{R}^{d}$. A classifier is a Borel-measurable map from $\mathfrak{X}$ to $\{-1,1\}$. For any classifier $h$, consider the two types of error probabilities,

$$
R^{-}(h):=\mathrm{P}(h(X) \geqslant 0 \mid Y=-1) \quad \text { and } \quad R^{+}(h):=\mathrm{P}(h(X)<0 \mid Y=1),
$$

and also the empirical counterpart

$$
\hat{R}^{-}(h):=\frac{1}{n^{-}} \sum_{i=1}^{n^{-}} 1\left(h\left(X_{i}^{-}\right) \geqslant 0\right)
$$

of $R^{-}(h)$, where $X_{1}, \ldots, X_{n^{-}}$is a (training) iid sample from the conditional distribution of $X$ given $Y=-1$, and $1(A)$ denotes the indicator of an assertion $A$ (so that $1(A)=1$ if $A$ is true and $1(A)=0$ if $A$ is false).

The mentioned result in [11] is as follows: there exist classifiers $h_{1}$ and $h_{2}$ and a probability distribution for $(X, Y)$ such that, for any $\alpha \in(0,1 / 2]$ and any r.v. $\Lambda$ with values in $[0,1]$ such that for the random "pseudo-classifier" $h_{\Lambda}:=\Lambda h_{1}+(1-\Lambda) h_{2}$ we have $\hat{R}^{-}\left(h_{\Lambda}\right)<\alpha$, the event that the "excess type II risk"

$$
R^{+}\left(h_{\Lambda}\right)-\min _{\lambda \in[0,1]: R^{+}\left(h_{\lambda}\right)} R^{+}\left(h_{\lambda}\right)
$$

is $\geqslant \alpha$ occurs with a probability $P \geqslant \min (\alpha, 1 / 4)$.

Using inequality (1) with condition $\sqrt{2}$ - instead of inequality $\sqrt{6}$ with condition (7), we can replace the conditions $\alpha \in(0,1 / 2]$ and $P \geqslant \min (\alpha, 1 / 4)$ in 
the cited result in [11] by the respective conditions $\alpha \in[c / n, 1]$ and $P \geqslant 1 / 4$, which will constitute a substantial improvement, in the case when $\alpha \geqslant c / n$. For the simpler case of $\alpha \in(0, c / n]$, an improvement over the result in [11 can be similarly obtained using Proposition 2.

\section{Proofs}

Here and in what follows,

$$
q:=1-p
$$

Proof of Theorem 1. If $n=1$, then

$$
\mathrm{P}(X>\mathrm{E} X)=\mathrm{P}(X>p)=\mathrm{P}(X=1)=p=n p \geqslant c>1 / 4,
$$

so that (1) holds, with the strict inequality.

Fix now any natural $n \geqslant 2$. Consider first the case when $c \leqslant n p<1$. Then

$$
\mathrm{P}(X>n p)=1-q^{n} \geqslant 1-q^{c / p}=1-\left(\frac{4}{3}\right)^{\frac{\ln (1-p)}{p}}>1-\left(\frac{4}{3}\right)^{-1}=\frac{1}{4},
$$

so that $\mathrm{P}(X>n p)>\frac{1}{4}$. Moreover, if $c=\ln \frac{4}{3}$ is replaced here by any $c_{1} \in(0, c)$, and if $p=c_{1} / n$ with $n \rightarrow \infty$, then $\mathrm{P}(X>n p)=1-q^{n}=1-\left(1-c_{1} / n\right)^{n} \rightarrow$ $1-e^{-c_{1}}<1-e^{-c}=1 / 4$.

Therefore, the constant factor $c$ in (2) cannot be improved and, moreover, without loss of generality (wlog)

$$
n p \geqslant 1
$$

So,

$$
m:=m_{n}:=\lfloor n p\rfloor+1 \in[2, n] .
$$

Introduce also

$$
p_{j}:=p_{n, j}:=\left(m_{n}-1\right) / j=(m-1) / j
$$

for $j \in\{m, \ldots, n\}$. Then

$$
\mathrm{P}(X>\mathrm{E} X)=\mathrm{P}\left(X_{n, p}>n p\right)=\mathrm{P}\left(X_{n, p} \geqslant m\right) \geqslant \mathrm{P}\left(X_{n, p_{n}} \geqslant m\right) .
$$

The latter inequality, which follows from the (strict) stochastic monotonicity of $X_{n, p}$ in $p$ and the inequality $p \geqslant p_{n}$, is strict unless $p=p_{n}$ (that is, unless $n p$ is an integer). Next, by part (i) of [10, Theorem 3] (which immediately follows from the second inequality in [2, Theorem 2.1], again by the stochastic monotonicity of $X_{n, p}$ in $\left.p\right)$, we have $\mathrm{P}\left(X_{j+1, p_{j+1}} \geqslant m\right)>\mathrm{P}\left(X_{j, p_{j}} \geqslant m\right)$ for all $j \in\{m, \ldots, n-1\}$. So, $\mathrm{P}\left(X_{n, p_{n}} \geqslant m\right) \geqslant \mathrm{P}\left(X_{m, p_{m}} \geqslant m\right)$, and this inequality is strict unless $m=n$. Also, $\mathrm{P}\left(X_{m, p_{m}} \geqslant m\right)=(1-1 / m)^{m} \geqslant(1-1 / 2)^{2}=1 / 4$, and $\mathrm{P}\left(X_{m, p_{m}}>m\right)>1 / 4$ unless $m=2$. It follows that $\mathrm{P}(X>\mathrm{E} X)>1 / 4$ unless $n=m=2$ and $n p$ is an integer. Thus, in view of $(10), \mathrm{P}(X>\mathrm{E} X)>1 / 4$ unless $n=2$ and $p=1 / 2$. That $\mathrm{P}(X>\mathrm{E} X)=1 / 4$ if $n=2$ and $p=1 / 2$ is trivial. This completes the proof of Theorem 1 . 
Proof of Proposition 2. If $c / n \geqslant p \geqslant 0$, then $\mathrm{P}(X>\mathrm{E} X)=1-(1-p)^{n}$. Next, $\left(1-(1-p)^{n}\right) /(n p)$ is decreasing in $p \in(0,1]$, so that for $p \in(0, c / n]$ we have $\left(1-(1-p)^{n}\right) /(n p) \geqslant\left(1-(1-c / n)^{n}\right) / c \geqslant\left(1-e^{-c}\right) / c=b$, so that $1-(1-p)^{n} \geqslant$ bnp. The inequality $1-(1-p)^{n} \geqslant p$ is obvious. This completes the proof of Proposition 2

\section{References}

[1] Alon, N., Hanneke, S., Holzman, R., Moran, S., 2021. A theory of PAC learnability of partial concept classes. arXiv:2107.08444. arXiv:2107.08444 [cs.LG].

[2] Anderson, T.W., Samuels, S.M., 1967. Some inequalities among binomial and Poisson probabilities, in: Proceedings of the Fifth Berkeley Symposium on Mathematical Statistics and Probability, Volume 1: Statistics, University of California Press, Berkeley, Calif.. pp. 1-12. URL: https://projecteuclid.org/euclid.bsmsp/1200512976

[3] Andonova Jaeger, S., 2005. Generalization bounds and complexities based on sparsity and clustering for convex combinations of functions from random classes. J. Mach. Learn. Res. 6, 307-340.

[4] Anthony, M., Shawe-Taylor, J., 1993. A result of Vapnik with applications. Discrete Appl. Math. 47, 207-217. doi 10.1016/0166-218X (93)90126-9.

[5] Greenberg, S., Mohri, M., 2014. Tight lower bound on the probability of a binomial exceeding its expectation. Statist. Probab. Lett. 86, 9198. URL: https://doi.org/10.1016/j.spl.2013.12.009, doi $10.1016 /$ j.spl.2013.12.009

[6] Haussler, D., 1992. Decision theoretic generalizations of the PAC model for neural net and other learning applications. Inf. Comput. 100, 78-150. URL: http://dx.doi.org/10.1016/0890-5401(92)90010-D, doi $10.1016 / 0890-5401(92) 90010-D$.

[7] Hoeffding, W., 1956. On the distribution of the number of successes in independent trials. Ann. Math. Statist. 27, 713-721. URL: https://doi. org/10.1214/aoms/1177728178, doi:10.1214/aoms/1177728178.

[8] Kontorovich, A., Pinelis, I., 2019. Exact lower bounds for the agnostic probably-approximately-correct (PAC) machine learning model. Ann. Statist. 47, 2822-2854. doi $10.1214 / 18-$ AOS1766.

[9] Korolev, V., Shevtsova, I., 2012. An improvement of the Berry-Esseen inequality with applications to Poisson and mixed Poisson random sums. Scand. Actuar. J., 81-105doi 10.1080/03461238.2010.485370.

[10] Pinelis, I., 2020. Monotonicity properties of the Poisson approximation to the binomial distribution. Statist. Probab. Lett. 167, 108901, 7. doi 10. $1016 / \mathrm{j} . \mathrm{spl} .2020 .108901$. 
[11] Rigollet, P., Tong, X., 2011. Neyman-Pearson classification, convexity and stochastic constraints. J. Mach. Learn. Res. 12, 2831-2855.

[12] Valiant, L.G., 1984. A theory of the learnable. Commun. ACM 27, 11341142 .

[13] Vapnik, V., 2006. Estimation of dependences based on empirical data. Information Science and Statistics, Springer, New York. Reprint of the 1982 edition, Afterword of 2006: Empirical inference science.

[14] Vapnik, V.N., 1998. Statistical learning theory. Adaptive and Learning Systems for Signal Processing, Communications, and Control, John Wiley \& Sons, Inc., New York. A Wiley-Interscience Publication.

\section{Appendix}

Second proof of Theorem 1. At least one of the following five cases must occur:

Case 1: $n p \geqslant 2$ and $n q \geqslant 2$ (recall the convention $q:=1-p$ in (8p)).

Case 2: $c \leqslant n p<1$ and $n \geqslant 1$, where $c$ is as in (3).

Case 3: $1 \leqslant n p<2$ and $n \geqslant 3$.

Case 4: $1<n q \leqslant 2$ and $n \geqslant 3$.

Case 5: $0<n q \leqslant 1$ and $n \geqslant 2$.

In particular, note that the cases when either (i) $n=1$ or (ii) $n=2$ and $p<1 / 2$ are covered by Case 2, whereas the case when $n=2$ and $p \geqslant 1 / 2$ is covered by Case 5 .

Consider now each of the five listed cases.

Case 1] The version of the Berry-Esseen bound given in [9, Theorem 1] implies

$$
\mathrm{P}(X>\mathrm{E} X)=\mathrm{P}(X>n p) \geqslant \frac{1}{2}-\varepsilon(n, p), \quad \text { where } \varepsilon(n, p):=\frac{c_{3}}{\sqrt{n}}\left(\frac{\rho}{\sigma^{3}}+c_{2}\right),
$$

$\rho=p^{3} q+q^{3} p, \sigma=\sqrt{p q}, c_{3}:=\frac{33477}{100000}, c_{2}=\frac{429}{1000}$.

Note that $p^{3} q / \sigma^{3}=p^{3 / 2}(1-p)^{-1 / 2}$ is convex in $p$ and, similarly, $q^{3} p / \sigma^{3}$ is convex in $p$, so that $\rho / \sigma^{3}$ and $\varepsilon(n, p)$ are convex in $p$. Therefore and in view of the Case 1 conditions $n p \geqslant 2$ and $n q \geqslant 2$, we have $\varepsilon(n, p) \leqslant \varepsilon(n, 2 / n)$ $=\varepsilon(n, 1-2 / n)=: \varepsilon_{*}(n)$, which is a simple algebraic function of $n$. For the derivative $\varepsilon_{*}^{\prime}(n)$ of $\varepsilon_{*}(n)$ in $n$, we see that $\varepsilon_{*}^{\prime}(n) n^{5 / 2}(n-2)^{3 / 2}$ is a polynomial in $(n-2)^{1 / 2}$, of degree 5 . Therefore, it is easy to see that $\varepsilon_{*}(n)$ is decreasing in $n \in[4,6]$, increasing in $n \in[7,89]$, and decreasing in $n \in[90, \infty)$. Also, the conditions $n p \geqslant 2$ and $n q \geqslant 2$ imply $n=n p+n q \geqslant 4$. So, in Case 1 . $\mathrm{P}(X>n p) \geqslant \frac{1}{2}-\max \left(\varepsilon_{*}(4), \varepsilon_{*}(89), \varepsilon_{*}(90)\right)>0.25587>1 / 4$.

Case 2. Then, by (9), $\mathrm{P}(X>n p)>\frac{1}{4}$. Moreover, it was shown in the paragraph containing (9) that the constant factor $c$ in (2) cannot be improved.

Case 3: Then

$$
\mathrm{P}(X>n p)=\mathrm{P}(X>1)=1-q^{n}-n q^{n-1} p,
$$


which is increasing in $p$, by the stochastic monotonicity of $X_{n, p}$ in $p$. So, wlog $p=1 / n$, in which case $\mathrm{P}(X>n p)=f_{3}(n):=1-(2-1 / n)(1-1 / n)^{n-1}$. The second derivative of $\ln \left(1-f_{3}(n)\right)$ is $1 /\left((2 n-1)^{2}(n-1) n\right)>0$, so that $\ln \left(1-f_{3}\right)$ is convex. Also, $\ln \left(1-f_{3}(n)\right) \rightarrow \ln (2 / e)$. Therefore, $\ln \left(1-f_{3}(n)\right)$ is decreasing (in $n \geqslant 3$ ) and $f_{3}(n)$ is increasing, from $f_{3}(3)=7 / 27>1 / 4$. Thus, $\mathrm{P}(X>n p)>\frac{1}{4}$ in Case 3 .

Case 4 Then $n-2 \leqslant n p<n-1, p \geqslant 1-2 / n$, and

$$
\mathrm{P}(X>n p)=\mathrm{P}(X \geqslant n-1)=f_{1}(p):=f_{1}(p, n):=p^{n}+n p^{n-1} q,
$$

and $f_{1}(p)$ is increasing in $p$, by the stochastic monotonicity of $X_{n, p}$ in $p$. Therefore, here wlog $p=1-2 / n$, and

$$
\tilde{f}_{1}(n):=f_{1}(1-2 / n, n)=\frac{3 n-2}{n-2}(1-2 / n)^{n} .
$$

Letting

$$
D \tilde{f}_{1}(n):=\tilde{f}_{1}^{\prime}(n) / \frac{(1-2 / n)^{n}(3 n-2)}{n-2}=\ln (1-2 / n)+\frac{6 n-8}{(n-2)(3 n-2)},
$$

we have

$$
\left(D \tilde{f}_{1}\right)^{\prime}(n)=-\frac{4\left(3 n^{2}-4 n+4\right)}{(3 n-2)^{2}(n-2)^{2} n}<0 .
$$

So, $D \tilde{f}_{1}$ is decreasing. Also, $D \tilde{f}_{1}(\infty-)=0$. It follows that $D \tilde{f}_{1}>0$ and hence $\tilde{f}_{1}$ is increasing, from $\tilde{f}_{1}(3)=\frac{7}{27}>\frac{1}{4}$. Thus, $\mathrm{P}(X>n p)>\frac{1}{4}$ in Case 4 .

Case 5 . Then $n>n p \geqslant n-1, p \geqslant 1-1 / n$, and hence

$$
\mathrm{P}(X>n p)=\mathrm{P}(X=n)=p^{n} \geqslant(1-1 / n)^{n},
$$

and $(1-1 / n)^{n}$ is increasing in $n \geqslant 2$, from $(1-1 / 2)^{2}=1 / 4$. So, $\mathrm{P}(X>n p)>\frac{1}{4}$ in Case 5 - except when $n=2$ and $p=1 / 2$, in which case $\mathrm{P}(X>n p)=\frac{1}{4}$.

This completes the second proof of Theorem 1 . 